\title{
Design and Implementation of the Road Traffic Offense Information Management System Based on J2EE
}

\author{
XIAO Ai-mei \\ Department of Computer Science and Technology, Shandong University of Technology Zibo, Shandong, China
}

\begin{abstract}
In recent years, the quantity of motor vehicles increases rapidly and the burden of the management of the road traffic are increasingly heavy. The information management of motor vehicles is disordered and the illegal information sharing of motor vehicles across the country is bad. Nowadays, in response to this mess, a B2B mode of the Road Traffic Offense Information Management System has been put forward. The system adopts SSH lightweight J2EE composite framework for development, and encapsulates such operations as complex algorithm, database interaction and data access through the JavaBeans component, which has enhanced the flexibility of the back-end data store and the promptness of the information analysis, and improved the practicability and scalability of the whole system. Since the system was put into practical operation, it has effectively improved the traffic police's regulatory capacity and efficiency in dealing with violations against traffic control and realized the sharing of motor vehicles' road traffic offense information nationwide. In the running process, the system is stable in performance, with good sharing of data and simple operation.
\end{abstract}

Key words: J2EE, Java bean, Oracle, road traffic offense, management information system.

\section{Introduction}

With the development of transportation in our country, the number of motor vehicle drivers and vehicles is growing. Accordingly, road traffic offense and accidents are increasing. At present, the traditional management mode has been still used on the aspects including the status of traffic violation, working condition of the police on duty and the adjustment of the location of the police on duty that for years. In recent years, with the rapidly expanding number of drivers and motor vehicles, the increasingly complex situation of road traffic, and the constantly updated vehicle performances and vehicle types, the traditional management method actually has been far from enough to satisfy the need of modern traffic management. To resolve a variety of existing issues effectively, present network resources should be taken full advantage of, which will be convenient for Traffic Police Department to implement scientific

Corresponding author: Xiao Ai-Mei, lecturer, research fields: algorithm and network information security. E-mail: xiao_am@163.com. management and establish a scientific and complete the Road Traffic Offense Information Management System for the sake of realizing the scientific and standardized administration of motor vehicle drivers' traffic violations and accident information.

The paper is organized as follows: Section 2 introduces the structure of system; Section 3 discusses system analysis and design overview; Section 4 contains detailed implementation of system; Section 5 contains the key technologies; Section 6 is the conclusion.

\section{The System Structure of J2EE}

J2EE is a technical criterion launched by SUN Company and formulated with the participation of many international mainstream manufactures. This criterion has the features of openness, standardization, structure, mutual manipulation and extensibility. The J2EE criterion has been widely accepted by the relevant industry. After many years of development, J2EE has actually become the standard of application servers. The typical four-layer structure of J2EE 
is:1)Client layer components that are running on the client machine;2)Web layer components that are running on the J2EE server;3)Business layer components that are running on the J2EE server;4)Enterprise information system layer software that is running on the EIS server[1, 2]. J2EE is divided into three tiers in general applications and the application of the three-tier framework is an extension of the standard client/server application framework [3].

\section{Overview Design}

3.1 The system mainly uses the following safety strategies:

(1) A network firewall professional is installed for campus network. It can effectively prevent the intrusion events; (2) The server security includes hardware and software. There may be a disk hardware damage. So the main hardware equipment of server can be replaced. The server can be backup termly. In terms of software, it ensures that the operating system hasn't loopholes and timing of antivirus; (3) The security of the database: In order to ensure the integrity of system data the system often does a database backup and prevents SQL injection, password brute force and so on; (4) Using the username and password login to realize the security of program. The system adopts different roles to different users access control[4].

\subsection{System analysis}

By analyzing and studying the characteristics of the road traffic offense and the decision management, the system decides to adopt the mode of $\mathrm{B} / \mathrm{S}$ (browser/server)[5]. Due to the extremely large quantity of system users, based on the detailed investigation of the existing commercialized database management system, the system adopts ORACLE 11g as the database management system. Oracle not only has excellent processing performance and reliable security assurance, but also can meet the requirement of large data volume storage; In the meanwhile, the Oracle Corporation has leading advantage in Java technology. Oracle $11 \mathrm{~g}$ provides strengthened function of development for the developers, which makes it easier for the developers to establish and deploy J2EE application [6].

\subsection{Overview design}

This system is carried out centering mainly on the driver, the traffic police and the decision-maker. And since this system is mainly about the management of three different kinds of traffic offense behaviors, it provides decision-making basis for the management department based on this. Through analyzing in detail the requirements of the topic and integrating all the requirements of the users, the overall business process of the system is pinned down as shown in Fig. 1.

The functions mainly conducted by the system are: (1) Information material record management function; (2) Traffic violations handling function; (3)Information analysis and decision support function; (4) Service work assessment for reward and punishment incentive function; (5) Strict law enforcement and security function; (6) Collaboration with related business such as vehicle management and accident function and so on.

To realize the above functions, we plan to break the system into seven modules: drivers' and vehicles' data switching module, road traffic offense information input and processing module, road traffic accident information input module, traffic offense and accidents in other provinces input module, data query module, calculation and analysis module, system maintenance and basic setting module.

\section{Detailed Implementation of System}

On account of the specific implementation, the system defines the main implementation functions of JavaBeans. The system first defines a number of packages and then on the basis of the division of function modules, defines a number of sub packages 
and a great deal of Bean. To realize the system's function, a total of seven packages are defined as follows: jtgl.tools (tools package), .road (road illegal information package), jtgl.watch (monitoring information package), jtgl.Accident (accident information package), jtgl.jsy (driver information package), jtgl.tjfx (statistical analysis package) and jtgl.sysSet (system setting package). The package jtgl.tools defines the database connection classes, system log classes and character string conversion classes; the package of jtgl.road defines the classes related with the road illegal information, mainly used for the entry and handling of the illegal information; jtgl.watch defines the category related to the monitoring of illegal handling which is used to handle the entry and processing of the monitoring information; jtgl.accident package is responsible for the entry and inquiry of accident information; jtgl.jsy serves drivers' query of vehicle information, driving license information and all kinds of traffic violation information. Only the design of jtgl.road is set as an example for explanation as follows.

The design and realization of traffic violations on road are included in the jtgl.road package, where jtgl.road. road input class mainly implements the input and processing functions of the road traffic offense information. This class involves many attributes and the relevant attribute is encapsulated in the corresponding class.

When entering the information, we except for several numbers on the disposition notice, all the relevant information should be fetched from the database. The system finishes this process by using the embedded codes in the JSP. When all the information is ready, the system will then use the java bean to invoke the method of insert () in the category of road Input to write into the database.

When dealing with illegal information, an operator inputs the number of notice and uses the defined method in the category of JavaBean and road deal to read detailed illegal information in the database. After finishing this, an operator also uses the method of update () in the category of road deal to save during the process of saving.

The function of validation on data input of users mainly relies on the javascript's script function of client-side. Judging before the users submit can save many error-checking codes in javabean, reduce server interaction and increase the response speed and working efficiency of the system.

\section{The Key Technologies}

The development of this system makes good use of Java object-oriented design ideas and gives play to the strong performance of the Oracle database. During the design process, the safe and complete management control mechanism has been adopted towards some key steps. The explanations for some key treatments during the developing process are illustrated as below.

\subsection{Daily log management}

Considering the requirements of the system safety are relatively high, the daily log is designed to track the user's operation all the time. The basic format of $\log$ is: date (accurate to second), user name, user IP, operation type and operation content. If it is the user who logins, no matter whether it is successful or not, the system will record this operation. The recorded operation type is "login", and the operation content is successful login or failed login. If it is failed login, there is a possibility of illicit attempt to log in by guessed passwords, so the wrong passwords should be recorded. Similar log management method is also adopted in other functional operations (especially modifications to records of illicit activities). Meanwhile, the Super Admin has daily log query function, which can select many modes such as date, category or user to check the system state and take steps against bad behaviors accordingly [7].

\subsection{Permissions setting}

There are many ways for authority management 
now, and the most common way is based on the role control. Or it can be described as classified and successive. A great number of systems adopt the user management mode which is similar to the current mainstream operation system. Users of a certain kind have a certain sort of jurisdiction. The greatest advantage of this kind of management mode is that it is convenient to manage. To grant setting permissions to a certain user, one only needs to classify him into a certain group (or category). Because of the various functions of this system, each operator may have different jurisdictions. Furthermore, they may add or delete some function jurisdiction for a user at any time according to the working demand. Therefore, the role control cannot be performed according to different roles at all. In order to solve this problem, a new mode is applied to the access control. The basic idea of the control strategy is to list all the functions in the system, then distribute a code to each function (such as $\mathrm{a}_{0}, \mathrm{a}_{1}$, $a_{2}, \ldots z_{9}$ ), and save the character string consisted of the user function code into session object after the user logs in the system to decide whether the user has the jurisdiction to execute this function. Demands can be satisfied well by adopting this model and it can assign permission for users randomly.

\subsection{The processing of chinese characters}

In the development of the JSP program, the phenomena of garbled Chinese characters are very common. One of the messy code situations appears when Chinese characters are directly output on the pages. This situation is relatively easy to be handled. In order to enable the JSP compiler to correctly decode JSP documents that contain Chinese characters, one only needs to designate the coded format in source files. The second condition is that the phenomenon of messy code also appears when data is transferred between pages using the post method. The way to address this phenomenon is to add the code instruction statement $<\%$ request.setCharacterEncoding(“gb2312”)\%> onto the page, which can solve the problem of messy code during the page transferring.

The extremely troublesome messy code problems are also met in data access because the database adopts the coding format of ISO-8859-1. The handling method is to convert the data into the format required by the database first when preserving it and then transform the format into GB2312 when displaying the data. In order to handle it conveniently, a conversion class named transString is defined in the jtgl.tools package and two methods defined in the class will implement the data format conversion. The methods are as follows:

package jtgl.tools;

public class transString

\{

public static String iso2gb(String str)

\{

try \{ str=new String(str.getBytes("ISO-8859-1"), "gb2312");

\}

catch( UnsupportEncodingException e)

\{

System.out.println("Encoding Error!");

\}

return str; \}

public static String gb2iso(String str) \{

try

$\{$ str=new

String(str.getBytes("gb2312"),"ISO-8859-1");

\}

catch(UnsupportEncodingException e)

\{

System.out.println("Encoding Error!");\} return str;

\}

\section{Conclusions}

The establishment of the system basically satisfies the requirements of the management of various traffic violation behaviors. The system development adopts 
B/S structure so that all services are conducted at the back-end, which overcomes the trouble of client software installation on the client machine; The J2EE platform occupies an important position in the current Web application development. The system adopts the popular J2EE technology development. Compared with other WEB programming technologies, J2EE has the advantage of high speed, safety, irrelevance with the platform and so on; The system adopts the multilayer structure system and makes use of JavaBean component to encapsulate such operations as complex algorithm, database interaction and data access through the JavaBean component, strengthening the saving flexibility of back-end data and rapidity of information analysis and improving the whole system's practicability and extensibility.

\section{References}

[1] Campione, M., and Walrath, K. 2001. The Java Tutorial: Object Oriented Programming or the Internet. Addison Wesley Longman, Inc..

[2] Kim, T. 2002. "Looking for a 3-tier App Builder." http://www.biztool.com/magazines/java/archives.

[3] Marinescu, F. 2002. Ejb Design Pattern, Advanced Patterns and Dioms. John Wiley \& Sons.

[4] Dr1di, F., Musehall, B., and Pernul, G. 2004. "Administration of an RBAC System[C], System Seienees.” In Proeeedings of the 37th Annual Hawaii International Conference on Publication.

[5] Inderjeet, S., Stearns, B., and Johnson, M. 2002. Designing Enterprise Applications with the J2EE Platform. MA: Addison-Wesley.

[6] Marinescu, F. 2003. Ejb Design Pattern, Advanced Patterns, Processes and Dioms. John Wiley\&Sons.

[7] Axis User's Guide [EB/OL] http://ws.apaehe.org/axis/java/user-guide.html .

Biography: Xiao Ai-mei, (1973-), F, lecturer, ShanDong University of Technology-College of Computer Science and Technology, research fields: algorithm and network information security. E-mail: xiao_am@163.com 